\title{
New integer-order approximations of discrete-time non-commensurate fractional-order systems using the cross Gramian
}

\author{
Marek Rydel $^{1}$ \\ Received: 15 January 2018 / Accepted: 29 August 2018 / \\ Published online: 8 September 2018 \\ (C) The Author(s) 2018
}

\begin{abstract}
This paper presents a new approach to modeling of linear time-invariant discrete-time non-commensurate fractional-order single-input single-output state space systems by means of the Balanced Truncation and Frequency Weighted model order reduction methods based on the cross Gramian. These reduction methods are applied to the specific rational (integer-order) FIR-based approximation to the fractional-order system, which enables to introduce simple, analytical formulae for determination of the cross Gramian of the system. This leads to significant decrease of computational burden in the reduction algorithm. As a result, a rational and relatively low-order state space approximator for the fractional-order system is obtained. A simulation experiment illustrates an efficiency of the introduced methodology in terms of high approximation accuracy and low time complexity of the proposed method.
\end{abstract}

Keywords Model order reduction - Cross Gramian · Frequency weighted · Non-commensurate fractional order system $\cdot$ FIR-based approximation

Mathematics Subject Classification (2010) 78M34 · 26A33 · 37M99

\section{Introduction}

Nowadays, fractional-order calculus, which is a generalization of ordinary derivation and integration to non integer orders, has been given considerable interest in various fields of research. This is due to the fact that many phenomena can be modeled more

\footnotetext{
Communicated by: Peter Benner

Marek Rydel

m.rydel@po.opole.pl
}

1 Department of Electrical, Control and Computer Engineering, Opole University of Technology, ul. Prószkowska 76, 45-758 Opole, Poland 
adequately by fractional-order models, such as heat conduction [19, 34, 55], electrochemical processes [11, ch. 2], [20, 51], biological systems [11, ch. 4], [30], or viscoelasticity problems [1, 42], [47, ch. 10.2]. In addition to the modeling problems, there has been an increase in research effort to apply the fractional calculus in control systems. Fractional-order versions of the PID controller, that is $P I^{\lambda} D^{\mu}$, have been found to produce a very good control performance [44, ch. 5-7], [48], as well as optimal/adaptive/predictive control strategies have been extended to fractional-order systems [15, 56, 64], [44, ch. 10].

The main problem occurred in fractional-order systems is their infinite time computational complexity, due to the fractional-order derivatives (or differences) incorporated in the model. Therefore, the key step in practical implementations is to approximate fractional-order systems by integer-order models having nearly the same properties in a given frequency range. A number of methods have been proposed in continuous- and discrete-time domains, using transfer function as well as state space representations, e.g., specific finite impulse response (FIR) and infinite impulse response (IIR) representations of fractional-order systems [7, 46, 58-60, 65]. However, an accurate approximation of the fractional-order system in a wide frequency range usually requires a very high integer-order approximator. Thus, to decrease the order of approximators, both in continuous- and discrete-time cases, various model reduction techniques have been proposed [21, 36, 41, 54, 60, 62].

Among reduction methods, a great attention has been given to the SVD-based methods, which use the balanced model realization theory. This concept enables an easy way of determination of the dominant part of the model and elimination of state variables which have negligible impact on the system properties. In general, the Balanced Truncation (BT) method consists in simultaneous diagonalization of the controllability $P$ and observability $Q$ Gramians [6, 23, 38, 45, 60]. A combination of the two, called the cross Gramian, which simultaneously encodes controllability and observability information on the system in a single matrix, can also be used in the model order reduction process. This concept was firstly introduced for single-input single-output (SISO) and symmetric multi-input multi-output (MIMO) systems [17, $18,39]$. Furthermore, this approach was also extended to nonlinear systems by the use of empirical Gramians [13, 24, 27].

The frequency weighted (FW) methods are extended versions of BT. They are based on either (1) direct application of input/output weighting functions $[16,29,63,66,68]$ or (2) Gramians in a restricted frequency interval $\Omega=\left[\omega_{1}, \omega_{2}\right]$ in the frequency domain $[5,10,22,28,31]$ or (3) Gramians in a restricted time interval $\tau=\left[t_{1}, t_{2}\right]$ in the time domain $[22,25,32,61]$. These lead to a higher model accuracy in selected frequency ranges, in particular when the parameters of the weighting functions and frequency/time intervals are optimized [50].

It is worth mentioning that the reduction process, in particular determination of Gramians for very high order systems, is highly time-consuming. This problem can be solved through the Fourier Model Reduction (FMR) method, which uses discrete-time Fourier coefficients to develop an intermediate order approximation of the original model [49, 60,67]. Controllability and observability Gramians can be calculated in an analytical way, which significantly reduces time complexity of the reduction process $[49,60]$. In this paper, new model order reduction methods 
combining the FIR-based approximation of a non-commensurate fractional-order system with BT and FW model order reduction methods based on the cross Gramian are proposed.

The remainder of the paper is organized as follows. A representation of noncommensurate fractional-order state space systems and integer-order approximation based on FIR technique are presented in Section 2. Consequently, this Section also gives the background for balancing the systems by use of the cross Gramian and recalls fundamentals of the BT and FW reduction methods for discrete-time state space systems. The main result in terms of new analytical formulae for determination of the cross Gramian for FIR-based approximation of the fractional-order system are presented in Section 3. Numerical examples of Section 4 confirm the effectiveness of the introduced methodology both in terms of modeling accuracy and low time complexity. Conclusions of Section 5 complete the paper.

\section{Preliminaries}

\subsection{System representation}

Consider a stable discrete-time non-commensurate fractional-order (NCFO) linear time-invariant (LTI) single-input single-output (SISO) state space system $G=\{A, B, C, D\}$

$$
\begin{array}{ll}
\Delta^{\alpha} x[k+1] & =A x[k]+B u[k], \quad x_{0}, \\
y[k] & =C x[k]+D u[k],
\end{array}
$$

where $k=0,1, \ldots, x[k] \in \mathfrak{R}^{n}$ is the state vector, $x_{0} \in \mathfrak{R}^{n}$ is the vector of initial conditions, $A \in \mathfrak{R}^{n \times n}, B \in \Re^{n \times 1}, C \in \mathfrak{R}^{1 \times n}, D \in \Re$ denote the system properties and $\Delta^{\alpha} x[k+1]$ is the fractional-order difference vector

$$
\Delta^{\alpha} x[k+1]=\left[\begin{array}{c}
\Delta^{\alpha_{1}} x_{1}[k+1] \\
\vdots \\
\Delta^{\alpha_{n}} x_{n}[k+1]
\end{array}\right],
$$

with $\alpha_{i} \in \Re$ being called the fractional (or non-integer) order, $\alpha_{i} \in(0,2)$ for $i=1, \ldots, n$.

Individual fractional-order differences $\Delta^{\alpha_{i}} x_{i}[k+1], i=1, \ldots, n$ can be represented by the well-known Grünwald-Letnikov fractional difference [44, ch. 3.5]

$$
\Delta^{\alpha_{i}} x_{i}[k+1]=\sum_{j=1}^{k+1}(-1)^{j}\left(\begin{array}{c}
\alpha_{i} \\
j
\end{array}\right) x_{i}(k-j+1), \quad k=0,1, \ldots,
$$

where $\left(\begin{array}{c}\alpha_{i} \\ j\end{array}\right)$ denotes the Newton binomial

$$
\left(\begin{array}{c}
\alpha_{i} \\
j
\end{array}\right)=\left\{\begin{array}{ll}
1 & j=0 \\
\frac{\alpha_{i}\left(\alpha_{i}-1\right) \cdots\left(\alpha_{i}-j+1\right)}{j !} & j>0
\end{array} .\right.
$$


In the case of $\alpha_{1}=\cdots=\alpha_{n}$, the system as in Eqn. (1) is called a commensurate fractional-order system.

\subsection{FIR-based approximation of discrete-time fractional-order system}

It is well known that the discrete-time integer- or fractional-order system can be described using the Fourier decomposition

$$
\mathcal{G}(z)=\sum_{k=0}^{\infty} g_{k} z^{-k},
$$

with $g_{k} \in \Re k=0,1, \ldots$, being the impulse response components for the system. In practice, the impulse response components usually approach values very close to zero and can be neglected after a certain implementation length $L$. In this way, a finite length approximation $\tilde{\mathcal{G}}_{L}(z)$ to the $\mathcal{G}(z)$ can be obtained. The error bounds corresponding to the introduced approximation

$$
\left\|\mathcal{G}(z)-\tilde{\mathcal{G}}_{L}(z)\right\|_{\infty}=\max _{|z|=1}\left|\mathcal{G}(z)-\tilde{\mathcal{G}}_{L}(z)\right|,
$$

usually decide on a value of the chosen implementation length $L$. However, the error bound given in Ref. [67] is not readily computable. Therefore, one way to determine the appropriate approximation length $L$ is to stop calculating Fourier components when their magnitudes $\left|g_{k}\right|$ decrease below a certain value [67].

The way of calculating Fourier components for a commensurate fractional-order system was proposed in Ref. [60] and it can be extended to NCFO systems as follows:

$$
g_{k}=\left\{\begin{array}{cl}
D & k=0 \\
C \phi_{k} & k=1,2, \ldots, L
\end{array},\right.
$$

where

$\phi_{k}=\left\{\begin{array}{cc}B & k=1 \\ \left(A+\operatorname{diag}\left(\alpha_{1}, \ldots, \alpha_{n}\right)\right) \phi_{k-1}-\sum_{j=2}^{k-1}(-1)^{j} \operatorname{diag}\left[\left(\begin{array}{c}\alpha_{1} \\ j\end{array}\right), \ldots,\left(\begin{array}{c}\alpha_{n} \\ j\end{array}\right)\right] \phi_{k-j} & k=2, \ldots, L\end{array}\right.$,

with $A, B, C, D$ and $\alpha_{i}$ as in Eqn. (1) and $L$ being the implementation length. This enables to present the FIR-based approximation of the system for the implementation length $L$ in state space form $\left(\tilde{G}_{L}=\{\tilde{A}, \tilde{B}, \tilde{C}, \tilde{D}\}\right)$ as follows:

$$
\begin{array}{ll}
\tilde{x}[k+1] & =\tilde{A} \tilde{x}[k]+\tilde{B} u[k], \\
\tilde{y}[k] & =\tilde{C} \tilde{x}[k]+\tilde{D} u[k],
\end{array}
$$

with $\tilde{A} \in \mathfrak{R}^{L \times L}, \tilde{B} \in \mathfrak{R}^{L \times 1}, \tilde{C} \in \mathfrak{R}^{1 \times L}$ and $\tilde{D} \in \mathfrak{R}$ defined as

$$
\tilde{A}=\left[\begin{array}{ccccc}
0 & 0 & 0 & \cdots & 0 \\
1 & 0 & 0 & \cdots & 0 \\
0 & 1 & 0 & \cdots & 0 \\
\vdots & \ddots & \ddots & \ddots & \vdots \\
0 & \cdots & 0 & 1 & 0
\end{array}\right], \quad \tilde{B}=\left[\begin{array}{c}
1 \\
0 \\
0 \\
\vdots \\
0
\end{array}\right], \quad \tilde{C}=\left[\begin{array}{llll}
g_{1} & g_{2} & \cdots & g_{L}
\end{array}\right], \quad \tilde{D}=g_{0}
$$


It is worth emphasizing that the state matrix $\tilde{A}$ is singular and nilpotent of order $L$.

Remark 1 For MIMO systems, Fourier components become matrices $g_{k} \in \Re^{n_{y} \times n_{u}}$, where $n_{u}$ and $n_{y}$ denote the numbers of inputs and outputs of the system, respectively. In that case, every element 0 and 1 in the state space form of the FIR-based approximation (6) changes into $0 \in \mathfrak{R}^{n_{u} \times n_{u}}$ and $I \in \mathfrak{R}^{n_{u} \times n_{u}}$ being the zero and identity matrices, respectively. Therefore, the order $\bar{L}$ of the FIR-based MIMO model depends on the number of inputs of the system and implementation length $\bar{L}=L n_{u}$ $[60,67]$.

\subsection{Cross Gramian-based model order reduction}

Balancing-related model order reduction methods can be interpreted as performing a linear state transformation $\bar{x} \rightarrow T \tilde{x}$. This concept is an easy way to determine a dominant part of the model which accurately describes the system dynamics. Transformation (or projection) matrix $T$ and its inverse are not unique. Nevertheless, the majority of algorithms determine its form based on the controllability $P$ and observability $Q$ Gramians of the system [23, 29, 37, 38, 49, 60, 63, 66].

Consider a SISO FIR-based approximation of the NCFO system as in Eqn. (6). Let $T$ be the transformation matrix of that system, which diagonalizes the system's controllability and observability Gramians, with decreasing Hankel singular values on the main diagonal

$$
T P T^{T}=T^{-T} Q T^{-1}=\operatorname{diag}\left(\sigma_{1}, \sigma_{2}, \ldots, \sigma_{r}, \sigma_{r+1}, \ldots, \sigma_{L}\right),
$$

where $\sigma_{1} \geq \sigma_{2} \geq \cdots \geq \sigma_{r}>\sigma_{r+1} \geq \cdots \geq \sigma_{L}>0$. Linear state transformation $\bar{x} \rightarrow T \tilde{x}$ enables to calculate the balanced form of the system $\bar{G}_{L}=\{\bar{A}, \bar{B}, \bar{C}, \bar{D}\}$ in the following way

$$
\bar{G}=\left[\begin{array}{c|c}
T \tilde{A} T^{-1} & T \tilde{B} \\
\hline \tilde{C} T^{-1} & \tilde{D}
\end{array}\right]=\left[\begin{array}{cc|c}
\bar{A}_{11} & \bar{A}_{12} & \bar{B}_{1} \\
\bar{A}_{21} & \bar{A}_{22} & \bar{B}_{2} \\
\hline \bar{C}_{1} & \bar{C}_{2} & \tilde{D}
\end{array}\right],
$$

where the submatrices $\tilde{A}_{r}=\bar{A}_{11} \in \Re^{r \times r}, \tilde{B}_{r}=\bar{B}_{1} \in \Re^{r \times 1}, \tilde{C}_{r}=\bar{C}_{1} \in \Re^{1 \times r}$ define the reduced-order state space model $\tilde{G}_{r}=\left\{\tilde{A}_{r}, \tilde{B}_{r}, \tilde{C}_{r}, \tilde{D}_{r}\right\}$ of order $r<L$.

Partitioning $T$ and $T^{-1}$ in the form of

$$
T=\left[\begin{array}{c}
T_{r} \\
T_{L-r}
\end{array}\right], \quad T^{-1}=\left[\begin{array}{cc}
T_{r}^{-1} & T_{L-r}^{-1}
\end{array}\right],
$$

where the matrices $T_{r} \in \mathfrak{R}^{r \times L}, T_{r}^{-1} \in \mathfrak{R}^{L \times r}$ such that $T_{r} T_{r}^{-1}=I_{r}$ are called truncation matrices and they are the only ones necessary to determine the reduced model [6, ch. 7.3] [63]

$$
\tilde{G}_{r}=\left[\begin{array}{c|c}
T_{r} \tilde{A} T_{r}^{-1} & T_{r} \tilde{B} \\
\hline \tilde{C} T_{r}^{-1} & \tilde{D}
\end{array}\right]
$$


The $\mathcal{H}_{\infty}$-norm of the difference between full $(L)$ and reduced $(r)$ order models is upper bounded by the twice of the sum of the neglected Hankel singular values [6, ch. 7.2]

$$
\left\|\tilde{G}_{L}-\tilde{G}_{r}\right\|_{\mathcal{H}_{\infty}} \leq 2 \sum_{i=r+1}^{L} \sigma_{i} .
$$

In addition to controllability and observability Gramians, another type of Gramian, known as the cross Gramian $\mathcal{X}$, can be used in the reduction process. The following definitions of reachability $\mathcal{R}(\tilde{A}, \tilde{B})$ and observability $\mathcal{O}(\tilde{C}, \tilde{A})$ matrices

$$
\mathcal{R}(\tilde{A}, \tilde{B})=\left[\begin{array}{cccc}
\tilde{B} & \tilde{A} \tilde{B} & \tilde{A}^{2} \tilde{B} & \cdots
\end{array}\right], \quad \mathcal{O}(\tilde{C}, \tilde{A})=\left[\begin{array}{c}
\tilde{C} \\
\tilde{C} \tilde{A} \\
\tilde{C} \tilde{A}^{2} \\
\vdots
\end{array}\right]
$$

can be a basis for the respective definitions of controllability, observability and cross Gramians [6, ch. 4.3.2]

$$
P=\mathcal{R} \mathcal{R}^{T}, \quad Q=\mathcal{O}^{T} \mathcal{O}, \quad \mathcal{X}=\mathcal{R O} .
$$

On this ground, for SISO and symmetric state-space MIMO systems, we can combine the three Gramians' definitions to obtain [18]

$$
\mathcal{X}^{2}=P Q
$$

As it is clearly seen from Eqn. (11), the cross Gramian simultaneously encodes controllability and observability properties into a single matrix $\mathcal{X}[17,18,39]$. For the considered SISO FIR-based approximation (6), due to the nilpotency of the state matrix $\tilde{A}$, the cross Gramian can be expressed as a finite sum

$$
\mathcal{X}=\sum_{k=0}^{L} \tilde{A}^{k} \tilde{B} \tilde{C} \tilde{A}^{k},
$$

and it is always a symmetric matrix for the SISO systems. The controllability and observability Gramians can be calculated from the respective discrete-time Lyapunov equations [6, ch. 4.3], [45], whereas the cross Gramian is determined from the following Sylvester equation [6, ch. 4.3.2], [17]

$$
\tilde{A} \mathcal{X} \tilde{A}-\mathcal{X}=-\tilde{B} \tilde{C} .
$$

In both cases, square roots of eigenvalues of the product of controllability and observability Gramians and absolute values of eigenvalues of the cross Gramian are input-output invariants and are equal to Hankel singular values of the model

$$
\sigma_{i}=\left|\lambda_{i}(\mathcal{X})\right|=\sqrt{\lambda_{i}(P Q)}
$$

For this reason, reduced model $\tilde{G}_{r}$ can be calculated on the basis of the cross Gramian, making this approach useful in decreasing computational complexity of the reduction algorithm, since solving two Lyapunov equations for $P$ and $Q$ is replaced by solving the single Sylvester equation only. 
Various methods can be used to obtain projection matrices from the cross Gramian $[4,5,9,27,57]$. The first approach can be performed on the basis of singular value decomposition (SVD) of the cross Gramian

$$
\mathcal{X}=U \Sigma V=\left(\begin{array}{ll}
U_{r} & U_{L-r}
\end{array}\right)\left(\begin{array}{cc}
\Sigma_{r} & 0 \\
0 & \Sigma_{L-r}
\end{array}\right)\left(\begin{array}{c}
V_{r} \\
V_{L-r}
\end{array}\right),
$$

where $U$ and $V$ are the unitary matrices, $U_{r} \in \mathfrak{R}^{L \times r}, V_{r} \in \mathfrak{R}^{r \times L}$. In the general case, the $\Sigma_{1}=\operatorname{diag}\left(\sigma_{1}^{c}, \ldots, \sigma_{r}^{c}\right)$ and $\Sigma_{2}=\operatorname{diag}\left(\sigma_{r+1}^{c}, \ldots, \sigma_{L}^{c}\right)$ matrices contain $\sigma_{i}^{c}$ sorted in decreasing order, which are the approximation to the Hankel singular values $\sigma_{i}$. In this way, only the approximation to the BT can be obtained [57]. However, for the SISO FIR-based model (6), the cross Gramian is a symmetric matrix, i.e., $\mathcal{X}=\mathcal{X}^{T}$ (see Eqn. (26)), which provide $\sigma_{i}^{c}=\left|\lambda_{i}(\mathcal{X})\right|=\sigma_{i}[6, \mathrm{ch}$. 3.2.3] and for that case the reduction is equivalent to the BT. The truncation matrices $T_{r}$ and $T_{r}^{-1}$ can be obtained through either the one-sided Galerkin projection

$$
T_{r}=U_{r}^{T}, \quad T_{r}^{-1}=U_{r},
$$

or the two-sided Petrov-Galerkin projection

$$
T_{r}=U_{r}^{T}, \quad T_{r}^{-1}=V_{r} .
$$

The second approach for determination of the transformation matrix $T$ relies on decomposition of matrix $\mathcal{X}$ into two blocks, which separate the strongly coupled from the weakly coupled controllable and observable states $[3,5,52]$

$$
T \mathcal{X} T^{-1}=\left(\begin{array}{cc}
S_{l} & 0 \\
0 & S_{s}
\end{array}\right)
$$

where eigenvalues of $S_{l} \in \mathfrak{R}^{r \times r}$ and $S_{S} \in \mathfrak{R}^{(L-r) \times(L-r)}$ are the large and small (in magnitude) Hankel singular values of the system.

One of the algorithms within the second approach requires of a block-ordered real Schur form of the cross Gramian and calculation of an additional Sylvester equation $[3,5,68]$

$$
\begin{gathered}
\mathcal{X}=\left(\begin{array}{ll}
V_{r} & V_{L-r}
\end{array}\right)\left(\begin{array}{cc}
S_{1} & S_{12} \\
0 & S_{2}
\end{array}\right)\left(\begin{array}{c}
V_{r}^{T} \\
V_{L-r}^{T}
\end{array}\right), \\
S_{1} \mathcal{Z}-\mathcal{Z} S_{2}=-S_{12},
\end{gathered}
$$

where $S_{1} \in \mathfrak{R}^{(r \times r)}, S_{2} \in \mathfrak{R}^{((L-r) \times(L-r))}$. This enables calculation of the truncation matrices as

$$
T_{r}=V_{r}^{T}-\mathcal{Z} V_{L-r}^{T}, \quad T_{r}^{-1}=V_{r} .
$$

Another algorithm within the second approach uses orthogonal Givens rotations in order to compute the ordered real Schur forms in ascending and descending orders of absolute eigenvalues along the diagonal [4, 52].

Taking into account that the algorithms based on the Schur decomposition are highly computationally involving, they can only be used for moderately complex models. This limits the maximum value of the implementation length $L$ of the FIR-based approximation to the fractional-order model. It finally affects the approximation accuracy of the obtained reduced order model, in particular for low-frequency 
range. On the other hand, algorithms for computing low-rank approximate solutions to the Sylvester equation, which provide the $r$-rank approximation to the cross Gramian $\mathcal{X}$, can be used in order to reduce computational complexity and enable approximate balancing reduction of large scale systems $[8,9,33,57]$.

Remark 2 The cross Gramian satisfies Eqn. (11) for SISO and symmetric MIMO systems only. Note that the cross Gramian for non-symmetric, square MIMO systems can be calculated from Eqn. (13), however without any theoretical background as for the symmetric case, so there is no guarantee for obtaining the reduced-order model of appropriate quality [9]. The approaches for non-square MIMO systems rely, e.g., on embedding the system into a symmetric square system by using a symmetrizer matrix [14], which produces a model of the same order but with more inputs and outputs [6, ch. 12.3], [57] or decompose the system into a set of individual SISO subsystems $[26,35,43,53]$.

\subsection{Frequency weighted model order reduction}

The aim of the reduction process based on the above discussed balancing method is system's approximation over all frequencies. In many cases, however, a good approximation is required in designated frequency ranges only. This leads to a specific frequency weighted balanced truncation. The FW method has been developed for stable models and stable input and output weighting functions with minimal realizations $W_{\text {in }}=\left\{A_{\text {in }}, B_{\text {in }}, C_{\text {in }}, D_{\text {in }}\right\}$ and $W_{\text {out }}=\left\{A_{\text {out }}, B_{\text {out }}, C_{\text {out }}, D_{\text {out }}\right\}$ of orders $m_{\text {in }}$ and $m_{\text {out }}$, respectively. It is well known that, in case of two-sided weighting, the controllability, observability, and cross Gramians are computed based on the system connected with the input weight $\left(\tilde{G}_{L} W_{i n}\right)$, the output weight $\left(W_{\text {out }} \tilde{G}_{L}\right)$, and both weights $\left(W_{\text {out }} \tilde{G}_{L} W_{i n}\right)$, respectively [6, ch. 7.6], [16, 29, 49, 63, 66, 68].

Remark 3 Note that for SISO systems, the input and output weighting functions can be very simply transformed into a single weighting function. Therefore, without the loss of generality for SISO systems, further examination will be conducted for onesided input weighting case only.

Accounting that no pole-zero cancellations occur during forming of $\tilde{G}_{L} W_{i n}$, the augmented system is given as follows [6, ch. 7.6], [29, 49, 63, 66, 68]

$$
\tilde{G}_{L} W_{i n}=\left[\begin{array}{c|c}
\tilde{A}_{i n} & \tilde{B}_{i n} \\
\hline \tilde{C}_{i n} & \tilde{D}_{i n}
\end{array}\right]=\left[\begin{array}{cc|c}
\tilde{A} & \tilde{B} C_{i n} & \tilde{B} D_{i n} \\
0 & A_{i n} & B_{i n} \\
\hline \tilde{C} & \tilde{D} C_{i n} & \tilde{D} D_{i n}
\end{array}\right],
$$

for which the cross Gramian is the solution of the following Sylvester equation

$$
\tilde{A}_{i n} \tilde{\mathcal{X}} \tilde{A}_{i n}-\tilde{\mathcal{X}}=-\tilde{B}_{i n} \tilde{C}_{i n} .
$$

It is important to emphasize that the application of the weighting functions affects the order of the solutions of the Lyapunov/Sylvester equations. However, in order to calculate truncation matrices $T_{r}$ and $T_{r}^{-1}$, Gramians of order $L$ are required. A number of algorithms to solve the problem have been developed [16, 29, 40, 63]. For 
instance, the cross Gramian of the augmented system $\tilde{G}_{L} W_{i n}$ can be partitioned as follows

$$
\tilde{\mathcal{X}}=\left[\begin{array}{ll}
\mathcal{X}_{11} & \mathcal{X}_{12} \\
\mathcal{X}_{21} & \mathcal{X}_{22}
\end{array}\right] \in \Re^{\left(L+m_{i n}\right) \times\left(L+m_{\text {in }}\right)}, \text { where } \mathcal{X}_{11} \in \Re^{L \times L}
$$

Like for the Enns technique [16] for frequency weighted controllability and observability Gramians, the frequency weighted cross Gramian $\overline{\mathcal{X}}$ can be defined as

$$
\overline{\mathcal{X}}=\mathcal{X}_{11},
$$

which means that the $\overline{\mathcal{X}}$ satisfy the following equation

$$
\tilde{A} \mathcal{X}_{11} \tilde{A}-\mathcal{X}_{11}=-\tilde{B} C_{i n} \mathcal{X}_{21} \tilde{A}-\tilde{B} D_{i n} \tilde{C} .
$$

A more general approach is based on the Schur complement of a matrix block, which is identical to the method proposed in [40, 63] for calculating frequency weighted controllability and observability Gramians

$$
\overline{\mathcal{X}}=\mathcal{X}_{11}-\gamma \mathcal{X}_{12} \mathcal{X}_{22}^{-1} \mathcal{X}_{21}
$$

where for $\gamma=0$, we have the Enns [16] and for $\gamma=1$ the Lin and Chiu [40] methods. In a general case, when $0<\gamma<1$, a combination of these two approaches is chosen [63].

Remark 4 All the subsequent steps of determination of the truncation matrices $T_{r}$ and $T_{r}^{-1}$ for the FW method are the same as for the methods presented in Section 2.3, with the frequency weighted cross Gramian $\overline{\mathcal{X}}$ substituted for $\mathcal{X}$.

\subsection{Fourier model reduction for continuous time systems}

The presented FMR cross Gramian model order reduction method can also be easily applied to reduction of continuous-time fractional-order systems. Note that the FIRbased approximation of the system and reduction algorithm are still realized in the $z$-domain. Therefore, approximation of continuous-time systems by the use of the considered methodology require the following steps:

1. Discretization of the continuous-time fractional-order system to its discrete-time counterpart.

2. Approximation of the discrete-time fractional-order system by the FIR-based model (see Section 2.2).

3. Reduction of the FIR-based model (see Sections 2.3 and 2.4).

4. Conversion of the reduced-order model back to the continuous-time domain.

Taking into account the above items, an additional step in terms of discretization of NCFO system has to be realized.

Consider a stable continuous-time non-commensurate fractional-order LTI SISO state space system

$$
\begin{aligned}
& \mathcal{D}^{\alpha} x(t)=A_{c} x(t)+B_{c} u(t), \\
& y(t)=C_{c} x(t)+D_{c} u(t),
\end{aligned}
$$


with $A_{c} \in \Re^{n \times n}, B_{c} \in \Re^{n \times 1}, C_{c} \in \Re^{1 \times n}, D_{c} \in \Re$ denoting the system properties and $\mathcal{D}^{\alpha} x(t)$ being the fractional-order derivative vector

$$
\mathcal{D}^{\alpha} x(t)=\left[\begin{array}{c}
\mathcal{D}^{\alpha_{1}} x_{1}(t) \\
\vdots \\
\mathcal{D}^{\alpha_{n}} x_{n}(t)
\end{array}\right] .
$$

Fractional-order derivatives $\mathcal{D}^{\alpha_{i}} x_{i}(t), i=1, \ldots, n$ can be represented by various definitions involving the Riemann-Liouville, Caputo or Grünwald-Letnikov derivatives [47, ch. 2]. Moreover, to obtain discrete-time non-commensurate fractionalorder system (1), various discretization schemes, such as Euler, Tustin or Al-Alaoui, can be used $[2,12]$. Taking into account that the simplest discretization procedure can be implemented by the use of the Euler operator to the Grünwald-Letnikov derivative, we arrive at [44, ch. 3.5]

$$
\left.\mathcal{D}^{\alpha_{i}} x(t)\right|_{t=k h} \approx\left[\frac{\Delta^{\alpha_{i}} x(k h)}{h^{\alpha_{i}}}\right],
$$

where $t=k h, k=0,1, \ldots$ denotes the (unnormalized) discrete-time and $h$ is the sampling interval. Therefore, transferring from continuous-time system (24) to its discrete-time counterpart (1) results in [44, ch. 3.5]

$$
A=h^{\alpha} A_{c}, \quad B=h^{\alpha} B_{c}, \quad C=C_{c}, \quad D=D_{c},
$$

with $A, B, C$, and $D$ being the matrices of the discrete-time NCFO system (1) and $h^{\alpha}=\operatorname{diag}\left(h^{\alpha_{1}}, \ldots, h^{\alpha_{n}}\right)$.

Taking into account that the reduced discrete-time model (8) is the integer-order model, a conversion process from the discrete to continuous domain can be obtained by using the well known classical methods, e.g., Tustin approach. A value of sampling period $h$ should be chosen with respect to an adequacy range of final reduced order model.

\section{Main result}

The main computational cost of the model reduction process based on the cross Gramian is the determination of the Gramian itself. Therefore, the main result of this paper is a new, analytical method for calculation of the cross Gramian of the FIR-based models.

\subsection{Cross Gramian for FIR-based approximation of NCFO system}

In general, the time complexity of calculating the cross Gramian by solving the Sylvester Eq. 13 is a class of cubic one. However, due to the special structure of the FIR-based approximation of discrete-time fractional-order model as in Eqn. (6), it is possible to determine an analytical solution of the cross Gramian. 
Lemma 1 Consider the FIR-based approximation (6) of a stable discrete-time noncommensurate fractional-order LTI SISO state space system (1). Then the cross Gramian for that model is an upper triangular Hankel matrix

$$
\mathcal{X}=\left[\begin{array}{ccccc}
g_{1} & g_{2} & \cdots & g_{L-1} & g_{L} \\
g_{2} & g_{3} & \cdots & g_{L} & 0 \\
\vdots & \vdots & \vdots & \vdots & \vdots \\
g_{L-1} & g_{L} & \cdots & 0 & 0 \\
g_{L} & 0 & \cdots & 0 & 0
\end{array}\right]
$$

where $g_{k}, k=1, \ldots, L$, are as in Eqn. (5).

Proof Because of the special structure of the state space for the FIR-based model, such that the matrix $\tilde{A}$ is a lower shift matrix, it results that $\tilde{A}^{k}$ is a k-th diagonal matrix and $\tilde{A}$ is nilpotent since $\tilde{A}^{L}=0$. Therefore, the reachability $\mathcal{R}(\tilde{A}, \tilde{B})$ and observability $\mathcal{O}(\tilde{C}, \tilde{A})$ matrices have finite dimensions ( $L$ columns and rows respectively) and are equal to

$$
\begin{aligned}
& \mathcal{R}(\tilde{A}, \tilde{B})=\left[\begin{array}{lllll}
\tilde{B} & \tilde{A} \tilde{B} & \tilde{A}^{2} \tilde{B} & \cdots & \tilde{A}^{L-1} \tilde{B}
\end{array}\right]=\left[\begin{array}{cccc}
1 & 0 & \cdots & 0 \\
0 & 1 & \cdots & 0 \\
\vdots & \vdots & \ddots & \vdots \\
0 & 0 & \cdots & 1
\end{array}\right], \\
& \mathcal{O}(\tilde{C}, \tilde{A})=\left[\begin{array}{c}
\tilde{C} \\
\tilde{C} \tilde{A} \\
\tilde{C} \tilde{A}^{2} \\
\vdots \\
C \tilde{A}^{L-1}
\end{array}\right]=\left[\begin{array}{cccc}
g_{1} & g_{2} & \cdots & g_{L} \\
g_{2} & g_{3} & \cdots & 0 \\
\vdots & \vdots & \ddots & \vdots \\
g_{L} & 0 & \cdots & 0
\end{array}\right] .
\end{aligned}
$$

Taking into account (10), we can determine the cross Gramian in a form of Eqn. (26). This completes the proof.

\subsection{Frequency weighted cross Gramian for FIR-based approximation of NCFO system with input weighting function}

The cross Gramian for the augmented system $\tilde{G}_{L} W_{i n}$ is a solution of the Sylvester Eq. 20. However, taking into account (19), the cross Gramian can be determined as a solution to four Sylvester equations of lower dimensions.

Lemma 2 Consider the discrete-time augmented system as in Eqn. (19). The cross Gramian in a form of Eqn. (21) can be determined as a solution to four consecutive Sylvester equations

$$
\begin{gathered}
A_{i n} \mathcal{X}_{21} \tilde{A}-\mathcal{X}_{21}=-B_{i n} \tilde{C}, \\
\tilde{A} \mathcal{X}_{11} \tilde{A}-\mathcal{X}_{11}=-\tilde{B} C_{i n} \mathcal{X}_{21} \tilde{A}-\tilde{B} D_{i n} \tilde{C} \\
A_{i n} \mathcal{X}_{22} A_{i n}-\mathcal{X}_{22}=-A_{i n} \mathcal{X}_{21} \tilde{B} C_{i n}-B_{i n} \tilde{D} C_{i n}, \\
\tilde{A} \mathcal{X}_{12} A_{i n}-\mathcal{X}_{12}=-\tilde{A} \mathcal{X}_{11} \tilde{B} C_{i n}-\tilde{B} C_{i n} \mathcal{X}_{21} \tilde{B} C_{i n}-\tilde{B} C_{i n} \mathcal{X}_{22} A_{i n}-\tilde{B} D_{i n} \tilde{D} C_{i n},
\end{gathered}
$$


where $\mathcal{X}_{11} \in \mathfrak{R}^{L \times L}, \mathcal{X}_{12} \in \mathfrak{R}^{L \times m_{\text {in }}}, \mathcal{X}_{21} \in \mathfrak{R}^{m_{\text {in }} \times L}, \mathcal{X}_{22} \in \mathfrak{R}^{m_{\text {in }} \times m_{\text {in }}}$.

Proof Combining Eqns. (19), (20), and (21), we obtain

$$
\left[\begin{array}{cc}
\tilde{A} & \tilde{B} C_{i n} \\
0 & A_{i n}
\end{array}\right]\left[\begin{array}{ll}
\mathcal{X}_{11} & \mathcal{X}_{12} \\
\mathcal{X}_{21} & \mathcal{X}_{22}
\end{array}\right]\left[\begin{array}{cc}
\tilde{A} & \tilde{B} C_{i n} \\
0 & A_{i n}
\end{array}\right]-\left[\begin{array}{cc}
\mathcal{X}_{11} & \mathcal{X}_{12} \\
\mathcal{X}_{21} & \mathcal{X}_{22}
\end{array}\right]=-\left[\begin{array}{cc}
\tilde{B} D_{i n} \tilde{C} & \tilde{B} D_{i n} \tilde{D} C_{i n} \\
B_{i n} \tilde{C} & B_{i n} \tilde{D} C_{i n}
\end{array}\right]
$$

Expanding the matrix description (31) into four submatrix equations, we arrive at Eqns. (27) to (30). This completes the proof.

Consider the input weighting function in terms of the FIR filter described as

$$
\mathcal{W}_{i n}(z)=\sum_{k=0}^{m_{i n}} w_{t} z^{-k}
$$

where $w_{k} \in \Re, k=0,1, \ldots, m_{i n}$ are the impulse response components of the weighting function (32). Then the augmented system $\tilde{G}_{L} W_{i n}$ as in Eqn. (19) consisting of the FIR-based approximation of the NCFO system (6) and input weighting function (32) can be presented in state space form as follows

$$
\begin{array}{ll}
\hat{x}[k+1] & =\tilde{A}_{i n} \hat{x}[k]+\tilde{B}_{i n} u[k], \\
\hat{y}[k] & =\tilde{C}_{i n} \hat{x}[k]+\tilde{D}_{i n} u[k],
\end{array}
$$

where $\tilde{A}_{\text {in }} \in \mathfrak{R}^{\left(L+m_{\text {in }}\right) \times\left(L+m_{\text {in }}\right)}, \tilde{B}_{\text {in }} \in \mathfrak{R}^{\left.\left(L+m_{\text {in }}\right) \times 1\right)}, \tilde{C}_{i n} \in \mathfrak{R}^{1 \times\left(L+m_{\text {in }}\right)}$ and $\tilde{D}_{\text {in }} \in \mathfrak{R}$ are defined as

$$
\begin{aligned}
& \tilde{A}_{i n}=\left[\begin{array}{c|c}
\tilde{A} & \tilde{B} C_{i n} \\
\hline 0 & A_{i n}
\end{array}\right]=\left[\begin{array}{ccccc|ccccc}
0 & 0 & 0 & \cdots & 0 & w_{1} & w_{2} & w_{3} & \cdots & w_{m_{i}} \\
1 & 0 & 0 & \cdots & 0 & 0 & 0 & 0 & \cdots & 0 \\
0 & 1 & 0 & \cdots & 0 & 0 & 0 & 0 & \cdots & 0 \\
\vdots & \ddots & \ddots & \ddots & \vdots & \vdots & \vdots & \vdots & \cdots & \vdots \\
0 & \cdots & 0 & 1 & 0 & 0 & 0 & 0 & \cdots & 0 \\
\hline 0 & 0 & 0 & \cdots & 0 & 0 & 0 & 0 & \cdots & 0 \\
0 & 0 & 0 & \cdots & 0 & 1 & 0 & 0 & \cdots & 0 \\
0 & 0 & 0 & \cdots & 0 & 0 & 1 & 0 & \cdots & 0 \\
\vdots & \vdots & \vdots & \vdots & \vdots & \vdots & \ddots & \ddots & \ddots & \vdots \\
0 & 0 & 0 & \cdots & 0 & 0 & \cdots & 0 & 1 & 0
\end{array}\right], \tilde{B}_{i n}=\left[\begin{array}{c}
\tilde{B} D_{i n} \\
\hline B_{i n}
\end{array}\right]=\left[\begin{array}{c}
w_{0} \\
0 \\
0 \\
\vdots \\
0 \\
\hline 1 \\
0 \\
0 \\
\vdots \\
0
\end{array}\right], \\
& \tilde{C}_{i n}=\left[\tilde{C} \mid \tilde{D} C_{i n}\right]=\left[\begin{array}{llllllll}
g_{1} & g_{2} & g_{3} & \cdots & g_{L} \mid g_{0} w_{1} & g_{0} w_{2} & \cdots & g_{0} w_{m_{i n}}
\end{array}\right], \tilde{D}_{i n}=\left[\tilde{D} D_{i n}\right]=g_{0} w_{0} \text {. }
\end{aligned}
$$

Due to the special structure of $\tilde{G}_{L} W_{\text {in }}$ given in Eqn. (33), it is possible to present a new, analytical method for determination of the cross Gramian for such a system.

Theorem 1 Consider the augmented system (33) consisting of the FIR-based approximation (6) of a stable discrete-time NCFO LTI SISO state space system (1) 
and the input weighting function (32). Then the cross Gramian for the augmented system is a block matrix such that

$$
\tilde{\mathcal{X}}=\left[\begin{array}{ll}
\mathcal{X}_{11} & \mathcal{X}_{12} \\
\mathcal{X}_{21} & \mathcal{X}_{22}
\end{array}\right]
$$

where

1) submatrix $\mathcal{X}_{11} \in \mathfrak{R}^{L \times L}$ is an upper triangular Hankel matrix

$$
\mathcal{X}_{11}=\left[\begin{array}{ccccc}
r_{1} & r_{2} & \cdots & r_{L-1} & r_{L} \\
r_{2} & r_{3} & \cdots & r_{L} & 0 \\
r_{3} & r_{4} & \cdots & 0 & 0 \\
\vdots & \vdots & \vdots & \vdots & \vdots \\
r_{L} & 0 & \cdots & 0 & 0
\end{array}\right]
$$

with

$$
r_{i}=\sum_{k=i}^{i+m_{i n}} w_{(k-i)} g_{k} \quad \text { for } i=1, \ldots, L,
$$

2) submatrix $\mathcal{X}_{12} \in \Re^{L \times m_{\text {in }}}$ is as follows

$$
\mathcal{X}_{12}=\left[\begin{array}{cccc}
\hat{r}_{1} & \hat{r}_{2} & \cdots & \hat{r}_{m_{i n}} \\
\hat{r}_{2,1} & \hat{r}_{2,2} & \cdots & \hat{r}_{2, m_{i n}} \\
\vdots & \vdots & \vdots & \vdots \\
\hat{r}_{L, 1} & \hat{r}_{L, 2} & \cdots & \hat{r}_{L, m_{i n}}
\end{array}\right]
$$

with

$$
\begin{array}{ll}
\hat{r}_{i}=\sum_{j=0}^{m_{i n}} w_{j} \sum_{k=0}^{j} g_{k} w_{(i+j-k)} & \text { for } i=1, \ldots, L, \\
\hat{r}_{i, j}=\hat{r}_{(i+j-1)}+\sum_{k=1}^{m_{i n}} r_{(i-k)} w_{(j+k-1)} & \text { for } i=1, \ldots, L, j=1, \ldots, m_{i n},
\end{array}
$$

3) submatrix $\mathcal{X}_{21} \in \mathfrak{R}^{m_{\text {in }} \times L}$ is a rectangular Hankel matrix

$$
\mathcal{X}_{21}=\left[\begin{array}{ccccc}
g_{1} & g_{2} & \cdots & g_{L-1} & g_{L} \\
g_{2} & g_{3} & \cdots & g_{L} & 0 \\
\vdots & \vdots & \vdots & . & \vdots \\
g_{m_{\text {in }}} & g_{m_{\text {in }}+1} & \cdots & 0 & 0
\end{array}\right]
$$

4) submatrix $\mathcal{X}_{22} \in \mathfrak{R}^{m_{\text {in }} \times m_{\text {in }}}$ is as follows

$$
\mathcal{X}_{22}=\left[\begin{array}{cccc}
\check{r}_{1,1} & \check{r}_{1,2} & \cdots & \check{r}_{1, m_{i n}} \\
\check{r}_{2,1} & \check{r}_{2,2} & \cdots & \check{r}_{2, m_{i n}} \\
\vdots & \vdots & \vdots & \vdots \\
\check{r}_{m_{i n}, 1} & \check{r}_{m_{i n}, 2} & \cdots & \check{r}_{m_{i n}, m_{i n}}
\end{array}\right],
$$


with

$$
\check{r}_{i, j}=\sum_{k=1}^{i} g_{(k-1)} w_{(j+i-k)} \quad \text { for } i=1, \ldots, m_{i n}, j=1, \ldots, m_{i n},
$$

and, for all the four items above, $g_{k}=0$ for $k>L, w_{k}=0$ for $k>m_{i n}, \hat{r}_{k}=0$ for $k<1$ or $k>L$.

Proof Consider the cross Gramian of the augmented system $\tilde{G}_{L} W_{\text {in }}$ partitioned as in Eqn. (34), where the submatrices of $\tilde{\mathcal{X}}$ can be concisely presented as follows:

$$
\mathcal{X}_{i j}=\left[\begin{array}{ccccc}
x_{1,1}^{i j} & x_{1,2}^{i j} & \cdots & x_{1,(\gamma-1)}^{i j} & x_{1, \gamma}^{i j} \\
x_{2,1}^{i j} & x_{2,2}^{i j} & \cdots & x_{2,(\gamma-1)}^{i j} & x_{2, \gamma}^{i j} \\
\vdots & \vdots & \vdots & \vdots & \vdots \\
x_{\beta, 1}^{i j} & x_{\beta, 2}^{i j} & \cdots & x_{\beta,(\gamma-1)}^{i j} & x_{\beta, \gamma}^{i j}
\end{array}\right],
$$

with the dimensions $\beta=\gamma=L$ for $\mathcal{X}_{11}, \beta=L, \gamma=m_{\text {in }}$ for $\mathcal{X}_{12}, \beta=m_{\text {in }}, \gamma=L$ for $\mathcal{X}_{21}$ and $\beta=\gamma=m_{\text {in }}$ for $\mathcal{X}_{22}$ resulting from Lemma 2.

Since $\tilde{A}$ and $A_{\text {in }}$ are lower shift matrices (compare Eqn. (33)), the left hand sides of the four consecutive Sylvester Eqs. 27 to (30) are as follows:

$$
-\left[\begin{array}{cccccc}
x_{1,1}^{i j} & x_{1,2}^{i j} & x_{1,3}^{i j} & \cdots & x_{1,(\gamma-1)}^{i j} & x_{1, \gamma}^{i j} \\
x_{2,1}^{i j}-x_{1,2}^{i j} & x_{2,2}^{i j}-x_{1,3}^{i j} & x_{2,3}^{i j}-x_{1,4}^{i j} & \cdots & x_{2,(\gamma-1)}^{i j}-x_{1, \gamma}^{i j} & x_{2, \gamma}^{i j} \\
x_{3,1}^{i j}-x_{2,2}^{i j} & x_{3,2}^{i j}-x_{2,3}^{i j} & x_{3,3}^{i j}-x_{2,4}^{i j} & \cdots & x_{3,(\gamma-1)}^{i j}-x_{2, \gamma}^{i j} & x_{3, \gamma}^{i j} \\
\vdots & \vdots & \vdots & \vdots & \vdots & \vdots \\
x_{\beta, 1}^{i j}-x_{(\beta-1), 2}^{i j} & x_{\beta, 2}^{i j}-x_{(\beta-1), 3}^{i j} & x_{\beta, 3}^{i j}-x_{(\beta-1), 4}^{i j} & \cdots & x_{\beta,(\gamma-1)}^{i j}-x_{(\beta-1), \gamma}^{i j} & x_{\beta, \gamma}^{i j}
\end{array}\right] .
$$

The right hand sides of the Sylvester Eqs. 27 and (28) are respectively equal to

$$
\begin{aligned}
& -B_{i n} \tilde{C}=\quad-\left[\begin{array}{ccccc}
g_{1} & g_{2} & \cdots & g_{L-1} & g_{L} \\
0 & 0 & \cdots & 0 & 0 \\
\vdots & \vdots & \vdots & \vdots & \vdots \\
0 & 0 & \cdots & 0 & 0
\end{array}\right], \\
& -\tilde{B} C_{i n} \mathcal{X}_{21} \tilde{A}-\tilde{B} D_{i n} \tilde{C}= \\
& -\left[\begin{array}{ccccc}
w_{0} g_{1}+\ldots+w_{m_{i n}} g_{\left(m_{i n}+1\right)} & w_{0} g_{2}+\ldots+w_{m_{i n}} g_{\left(m_{i n}+2\right)} & \cdots & w_{0} g_{(L-1)}+w_{1} g_{L} & w_{0} g_{L} \\
0 & 0 & \cdots & 0 & 0 \\
\vdots & \vdots & \vdots & \vdots & \vdots \\
0 & 0 & \cdots & 0 & 0
\end{array}\right] \text {. }
\end{aligned}
$$

Both above matrices contain nonzero entries in the first rows only, which proves that $\mathcal{X}_{11}$ and $\mathcal{X}_{21}$ are the upper triangular Hankel matrix and rectangular Hankel matrix, respectively. 
The right hand side of the Sylvester Eq. 29 can be presented as (compare Eqn. (33))

$$
\begin{aligned}
-A_{i n} \mathcal{X}_{21} \tilde{B} C_{i n}- & B_{i n} \tilde{D} C_{i n}= \\
- & {\left[\begin{array}{ccccc}
g_{0} w_{1} & g_{0} w_{2} & \cdots & g_{0} w_{\left(m_{i n}-1\right)} & g_{0} w_{m_{i n}} \\
g_{1} w_{1} & g_{1} w_{2} & \cdots & g_{1} w_{\left(m_{i n}-1\right)} & g_{1} w_{m_{i n}} \\
\vdots & \vdots & \vdots & \vdots & \vdots \\
g_{\left(m_{i n}-1\right)} w_{1} & g_{\left(m_{i n}-1\right)} w_{2} & \cdots & g_{\left(m_{i n}-1\right)} w_{\left(m_{i n}-1\right)} & g_{\left(m_{i n}-1\right)} w_{m_{i n}}
\end{array}\right] . }
\end{aligned}
$$

After simple manipulations, we arrive at

$$
\mathcal{X}_{22}=\left[\begin{array}{ccccc}
g_{0} w_{1} & g_{0} w_{2} & \cdots & g_{0} w_{\left(m_{i n}-1\right)} & g_{0} w_{m_{\text {in }}} \\
g_{1} w_{1}+x_{1,2}^{22} & g_{1} w_{2}+x_{1,3}^{22} & \cdots & g_{1} w_{\left(m_{\text {in }}-1\right)}+x_{1, m_{i n}}^{22} & g_{1} w_{m_{\text {in }}} \\
\vdots & \vdots & \vdots & \vdots & \vdots \\
g_{\left(m_{\text {in }}-1\right)} w_{1} & g_{\left(m_{\text {in }}-1\right)} w_{2} & \cdots & g_{\left(m_{\text {in }}-1\right)} w_{\left(m_{\text {in }}-1\right)} & g_{\left(m_{\text {in }}-1\right)} w_{m_{\text {in }}} \\
+x_{\left(m_{\text {in }}-1\right), 2}^{22} & +x_{\left(m_{\text {in }}-1\right), 3}^{22} & & +x_{\left(m_{\text {in }}-1\right), m_{\text {in }}}^{22} &
\end{array}\right] .
$$

Similar elementary operations performed on the right hand side of the Sylvester Eq. 30 can lead to

$$
\mathcal{X}_{12}=\left[\begin{array}{ccccc}
\sigma_{1} & \sigma_{2} & \cdots & \sigma_{\left(m_{i n}-1\right)} & \sigma_{m_{i n}} \\
r_{1} w_{1}+x_{1,2}^{12} & r_{1} w_{2}+x_{1,3}^{12} & \cdots & r_{1} w_{m_{\text {in }}-1}+x_{1, m_{i n}}^{12} & r_{1} w_{m_{\text {in }}} \\
r_{2} w_{1}+x_{2,2}^{12} & r_{2} w_{2}+x_{2,3}^{12} & \cdots & r_{2} w_{m_{i n}-1}+x_{2, m_{i n}}^{12} & r_{2} w_{m_{\text {in }}} \\
\vdots & \vdots & \vdots & \vdots & \vdots \\
r_{(L-1)} w_{1} & r_{(L-1)} w_{2} & \cdots & r_{(L-1)} w_{\left(m_{i n}-1\right)} & r_{(L-1)} w_{m_{i n}} \\
+x_{(L-1), 2}^{12} & +x_{(L-1), 3}^{12} & & +x_{(L-1), m_{i n}}^{12} &
\end{array}\right],
$$

where

$$
\begin{aligned}
\sigma_{0} & =g_{1} w_{1}+\cdots+g_{m_{i n}} w_{m_{i n}}, \\
\sigma_{1} & =w_{1} \sigma_{0}+w_{1} \check{r}_{1,2}+\cdots+w_{m_{i n}} \check{r}_{m_{i n}, 2}+w_{0} g_{0} w_{1}, \\
\sigma_{2} & =w_{2} \sigma_{0}+w_{1} \check{r}_{1,3}+\cdots+w_{m_{i n}} \check{r}_{m_{i n}, 3}+w_{0} g_{0} w_{2}, \\
\vdots & \\
\sigma_{\left(m_{\text {in }}-1\right)} & =w_{\left(m_{i n}-1\right)} \sigma_{0}+w_{1} \check{r}_{1, m_{i n}}+\cdots+w_{m_{i n}} \check{r}_{m_{i n}, m_{i n}}+w_{0} g_{0} w_{\left(m_{i n}-1\right)}, \\
\sigma_{m_{i n}} & =w_{m_{\text {in }}} \sigma_{0}+w_{0} g_{0} w_{m_{i n} .} .
\end{aligned}
$$

Accounting for the elements $x_{i, j}^{12}$ and $x_{i, j}^{22}$ occurring in the previous rows, e.g., starting from $x_{1,2}^{12}=\sigma_{2}$ in $\mathcal{X}_{12}$ and from $x_{1,2}^{22}=g_{0} w_{2}$ in $\mathcal{X}_{22}$, we arrive at Eqns. (36) and (38). This completes the proof.

As mentioned above, the cross Gramian for the augmented system, consisting of the FIR-based approximation for NCFO system and input weighting function, satisfies (20) for SISO systems only. Note that the cross Gramian for non-symmetric square MIMO systems can be determined using Theorem 1 with substitution of all the elements $g_{k}, w_{k}$ by the respective matrices $g_{k} \in \Re^{n_{u} \times n_{u}}, w_{k} \in \Re^{n_{u} \times n_{u}}$ and all the elements 0 and 1 by the respective zero and identity matrices $0 \in \Re^{n_{u} \times n_{u}}$ and $I \in \Re^{n_{u} \times n_{u}}$, where $n_{u}$ denotes both the number of inputs and outputs of the system. 
However, for non-symmetric square MIMO systems the singular values of the cross Gramian $\sigma_{i}^{c}$ (14) are only the approximation to the Hankel singular values $\sigma_{i}$ [57]. Therefore, it must be emphasized that without any theoretical background as for the SISO case, there is no guarantee for obtaining the reduced-order model of appropriate quality.

\subsection{Frequency weighted Fourier model reduction algorithm}

The frequency weighted Fourier model reduction (FMR-FW) algorithm for reduction of fractional-order systems can be summarized in the following steps:

1. For continuous-time NCFO system, use discretization procedure to obtain discrete-time counterpart as in Eqn. (25).

2. Calculate $g_{k}$ components for NCFO system using Eqn. (5).

3. Specify $w_{k}$ components of the input weighting function (32).

4. Create the augmented system $\tilde{G}_{L} W_{i n}$ as in Eqn. (33), consisting of the FIR-based approximation of NFCO system and input weighting function.

5. Use Theorem 1 to analytically calculate the cross Gramian $\tilde{\mathcal{X}}$ of the system $\tilde{G}_{L} W_{i n}$.

6. Select coefficient $\gamma$ and calculate frequency weighted cross Gramian $\overline{\mathcal{X}}$ based on Eqn. (23).

7. Calculate the truncation matrices $T_{r}$ and $T_{r}^{-1}$ using either the one-sided Galerkin projection (15) or the two-sided Petrov-Galerkin projection (16) or the algorithm based on the Schur decomposition (18).

8. Reduce order of the model as in Eqn. (8).

9. Use $\tilde{G}_{r}$ as a final model for the discrete-time NCFO system or convert $\tilde{G}_{r}$ to the continuous-time domain using classical methods (e.g., Tustin approach) in order to obtain a final model for the continuous-time NCFO system.

\section{Simulation examples}

The effectiveness of the reduction process by the use of the introduced methodology will be demonstrated on test examples. All the computations were done on a board 2 IntelXeon E5-2683V3 CPUs with 2.0 GHz clock and 128 GB RAM using MATLAB/Simulink 2015b software.

Example 1 Consider the discrete-time NCFO state space system (1) with

$$
\left[\begin{array}{c|c}
A & B \\
\hline C & D
\end{array}\right]=\left[\begin{array}{cccc|c}
-2.2 & -1.5 & -0.45 & -0.05 & 1 \\
1 & 0 & 0 & 0 & 0 \\
0 & 1 & 0 & 0 & 0 \\
0 & 0 & 1 & 0 & 0 \\
\hline 0 & 1 & 0 & 0 & 0
\end{array}\right], \quad \Delta^{\alpha}=\left[\begin{array}{c}
\Delta^{0.8} \\
\Delta^{0.4} \\
\Delta^{0.5} \\
\Delta^{0.25}
\end{array}\right] .
$$

The system is approximated by the FIR-based models (6), with the $\mathcal{H}_{\infty}$-norm approximation errors equal to $0.0855,0.0204,0.0044$ for the respective implementation 
lengths $L$ being 100, 1000, and 10000. Hankel singular values for all models are presented in Fig. 1a. Figure $1 \mathrm{~b}$ shows approximation errors, the upper bound of $\mathcal{H}_{\infty^{-}}$ norm (9) calculated on the basis of HSV for FIR-based models and the actual value of $\mathcal{H}_{\infty}$-norm for the reduced models obtained by use of the FMR-BT method. The results show that approximation accuracy for the FIR-based model plays a crucial role in the reduction process as the global approximation error for the reduced model is a sum of errors for the FIR-based approximation and the reduction process.

Example 2 Consider the NFCO system as in Example 1 approximated by the FIRbased model (6), with the implementation length $L=10^{4}$. The input frequency weighting function is applied in the form of a low-pass FIR digital filter with cutoff frequency $\omega_{c}=0.01 \pi[\mathrm{rad} / \mathrm{s}]$ and $m_{i n}=12$. The system is reduced to models of order $r=4$ using the FMR-BT method based on the cross Gramian given as in Lemma 1 as well as the FMR-FW method based on the frequency weighted cross Gramian as in Eqn. (23) for $\gamma=0,0.35,0.7,1.0$ and the one-sided Galerkin projection for determination of the truncation matrices $T_{r}$ and $T_{r}^{-1}$. The cross Gramian of the augmented system $\tilde{\mathcal{X}}$ is calculated based on Theorem 1 . Since the matrix $\mathcal{X}_{22}$ may be ill-conditioned, the Moore-Penrose pseudoinverse can be used. The frequency responses of NCFO system, high integer-order FIR-based approximation and reduced models, as well as the approximation errors are presented in Fig. 2.

Example 3 Consider the same example of fractional-order system and the FMR-FW algorithm as in Example 2. Figure 3 shows the approximation errors of reduced models obtained by the use two algorithms of determination for truncation matrices $T_{r}$ and $T_{r}^{-1}$, that is (1) the one-sided Galerkin projection (15), denoted as FMR-FW-G and (2) the algorithm based on the Schur decomposition (18), denoted as FMR-FW$\mathrm{S}$. Table 1 presents the values of the approximation errors for the analyzed models in terms of $D C E$ - steady-state approximation error, $M S E$ - mean square approximation error for the frequency characteristics in the frequency range $\omega \in\left[10^{-5}, \pi\right]$ and $\mathcal{H}_{\infty}$-norm approximation error.

a

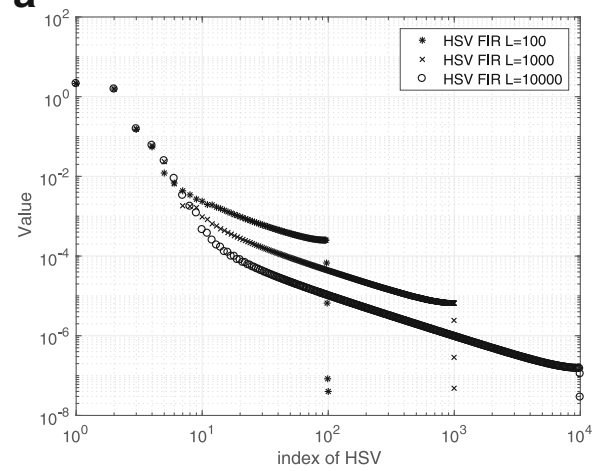

b

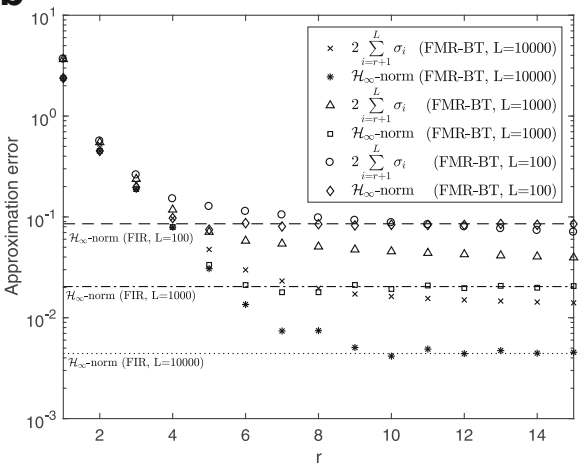

Fig. 1 a Hankel singular values of FIR-based models. b $\mathcal{H}_{\infty}$-norm errors for the reduced models 

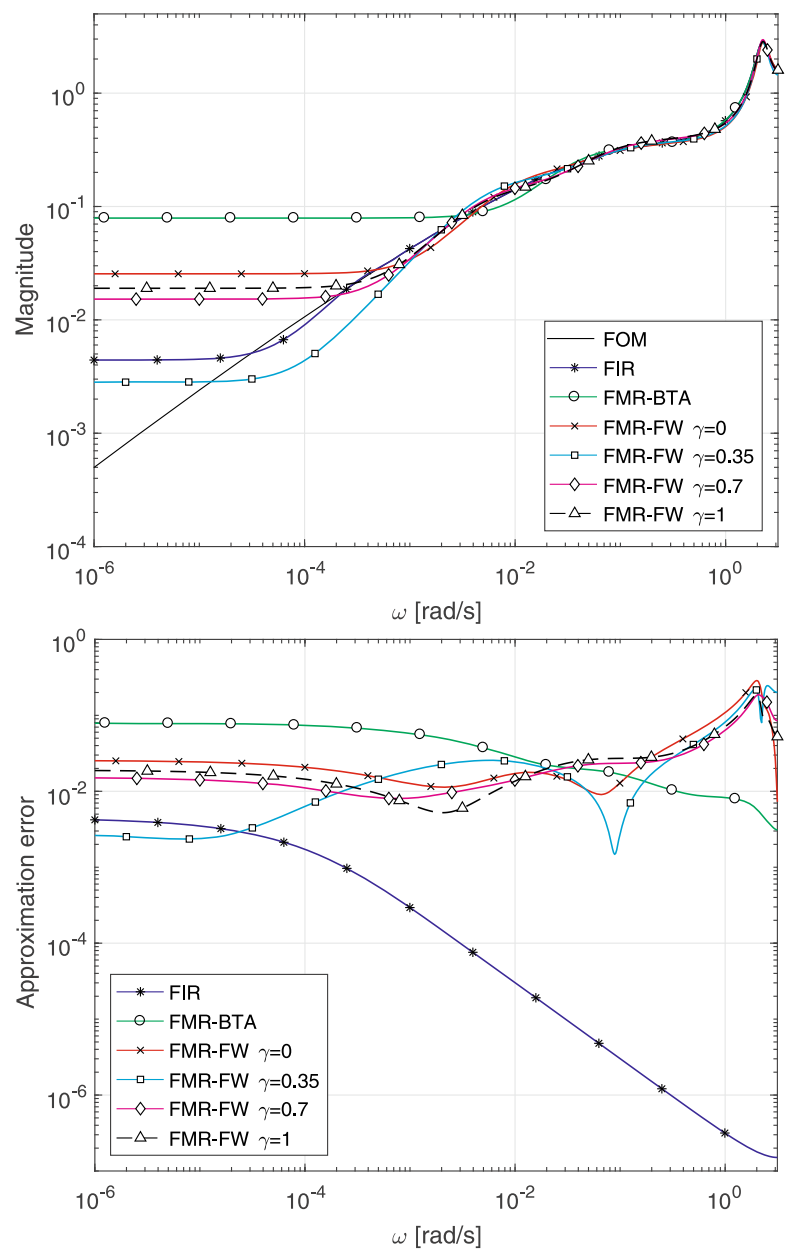

Fig. 2 Frequency responses and approximation errors for the reduced models

To compare time complexities of (each iteration of) the FMR-FW algorithm the fractional-order system was reduced for various implementation lengths $L$ and various orders $m_{i n}$ of the input weighting function. Table 2 shows times required for determination of the augmented system (33), item 1 in the table, calculation of the cross Gramian from the Sylvester Eq. 20, item 2, calculation of the cross Gramian based on Lemma 2, item 3, calculation of the cross Gramian based on Theorem 1, item 4, calculation of truncation matrices $T_{r}$ and $T_{r}^{-1}$ based on the one-sided Galerkin projection (15), item 5, calculation of truncation matrices based on the real Schur decomposition (18), item 6.

The results presented in Figs. 2 and 3 and Table 1 show that FMR-FW gives a good approximation accuracy, in particular for low frequency range, in comparison 


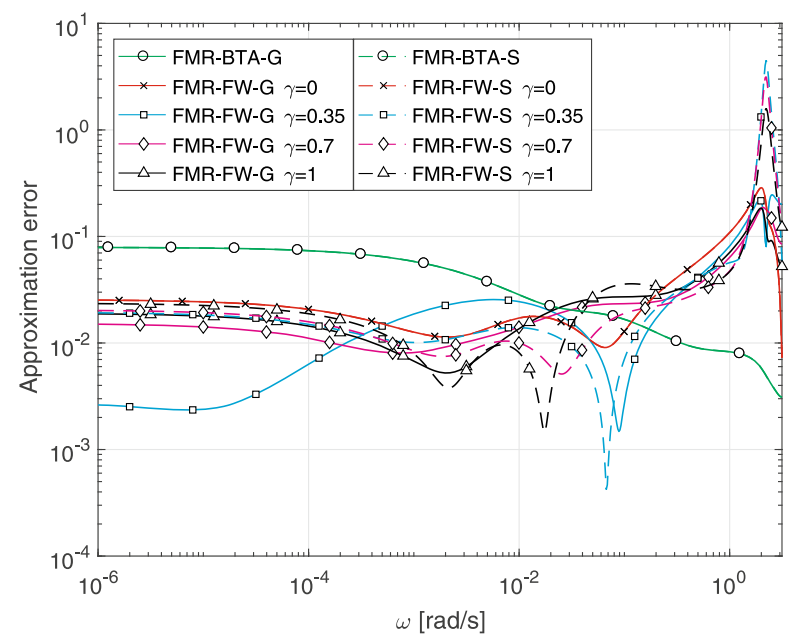

Fig. 3 Approximation errors for the reduced models

to the FMR-BT method, due to application of the low-pass filter. It can be seen that the approximation error in the low frequency range is in a large part the result of the FIR-based approximation of the NCFO system. Reduced order models obtained by the use of both methods for determination of truncation matrices give very similar results. Taking into account that the algorithm based on the Schur decomposition is much more computationally involving, it is strongly recommended to use the onesided Galerkin projection. It can also be seen from Table 2 that the computational times for the FMR-FW algorithm using the introduced analytical solution to the cross Gramian and the one-sided Galerkin projection is the least time consuming.

The Matlab scripts used to compute the presented results can be obtained from: http://doi.org/10.5281/zenodo.1256699.

Table 1 Approximation errors for models

\begin{tabular}{llll}
\hline & $D C E$ & $M S E$ & $\mathcal{H}_{\infty}$-norm \\
\hline FIR & $4.4 \mathrm{e}-3$ & 0.1003 & $4.4 \mathrm{e}-3$ \\
FMR-BT & $79.0 \mathrm{e}-3$ & 62.72 & 0.079 \\
FMR-FW-G $(\gamma=0)$ & $25.5 \mathrm{e}-3$ & 5.852 & 0.2864 \\
FMR-FW-S $(\gamma=0)$ & $25.5 \mathrm{e}-3$ & 5.852 & 0.2864 \\
FMR-FW-G $(\gamma=0.35)$ & $2.80 \mathrm{e}-3$ & 0.485 & 0.2459 \\
FMR-FW-S $(\gamma=0.35)$ & $19.4 \mathrm{e}-3$ & 3.283 & 4.5017 \\
FMR-FW-G $(\gamma=0.7)$ & $15.2 \mathrm{e}-3$ & 1.913 & 0.1872 \\
FMR-FW-S $(\gamma=0.7)$ & $20.4 \mathrm{e}-3$ & 3.627 & 3.1588 \\
FMR-FW-G $(\gamma=1)$ & $19.0 \mathrm{e}-3$ & 3.087 & 0.1846 \\
FMR-FW-S $(\gamma=1)$ & $23.6 \mathrm{e}-3$ & 4.960 & 1.5921 \\
\hline
\end{tabular}


Table 2 Computation time (in seconds) of the FMR-FW algorithm

\begin{tabular}{|c|c|c|c|c|c|c|}
\hline & & $L=100$ & $L=300$ & $L=1000$ & $L=3000$ & $L=10000$ \\
\hline \multirow[t]{2}{*}{ 1) } & $m_{i}=10$ & $23.23 \mathrm{e}-3$ & $184.0 \mathrm{e}-3$ & 1.938 & 16.89 & 185.7 \\
\hline & $m_{i}=100$ & $41.66 \mathrm{e}-3$ & $202.3 e-3$ & 2.092 & 18.31 & 201.7 \\
\hline \multirow[t]{2}{*}{ 2) } & $m_{i}=10$ & $7.023 e-3$ & $93.79 \mathrm{e}-3$ & 2.712 & 163.5 & 7028 \\
\hline & $m_{i}=100$ & $43.80 \mathrm{e}-3$ & $199.6 e-3$ & 3.834 & 175.8 & 7123 \\
\hline \multirow[t]{2}{*}{ 3) } & $m_{i}=10$ & $9.892 \mathrm{e}-3$ & $103.7 \mathrm{e}-3$ & 2.907 & 170.1 & 7271 \\
\hline & $m_{i}=100$ & $33.50 \mathrm{e}-3$ & $141.5 \mathrm{e}-3$ & 3.139 & 173.7 & 7389 \\
\hline \multirow[t]{2}{*}{ 4) } & $m_{i}=10$ & $0.771 \mathrm{e}-3$ & $2.022 \mathrm{e}-3$ & 0.0330 & 0.4871 & 4.575 \\
\hline & $m_{i}=100$ & $2.561 \mathrm{e}-3$ & $3.943 \mathrm{e}-3$ & 0.0342 & 0.4759 & 4.551 \\
\hline 5) & & $2.754 \mathrm{e}-3$ & $20.20 \mathrm{e}-3$ & 0.1693 & 2.194 & 24.74 \\
\hline 6) & & $46.77 \mathrm{e}-3$ & $689.0 \mathrm{e}-3$ & 22.11 & 617.9 & 23941 \\
\hline
\end{tabular}

\section{Conclusions}

In this paper, a new approximation methodology for modeling of discrete-time non-commensurate fractional-order state space systems by integer-order state-space models has been offered. The introduced FW reduction method is based on cross Gramian of a high-order FIR-based approximation to the fractional-order system. The main contribution of the paper is the introduction of analytical formulae enabling low-cost calculation of the cross Gramians, including the frequency weighted one, instead of a very time consuming process of classical solving the Sylvester equation. Simulation examples illustrate the efficiency of the proposed methodology, both in terms of high accuracy within prespecified frequency ranges and low computation effort of the reduction algorithms.

Acknowledgements The author is indebted to Prof. Krzysztof J. Latawiec and Prof. Rafał Stanisławski for their stimulating discussions and to anonymous reviewers for their instructive comments.

Funding information This work was supported by the Polish National Science Centre on the base of decision no. DEC-2017/01/X/ST7/00885.

Open Access This article is distributed under the terms of the Creative Commons Attribution 4.0 International License (http://creativecommons.org/licenses/by/4.0/), which permits unrestricted use, distribution, and reproduction in any medium, provided you give appropriate credit to the original author(s) and the source, provide a link to the Creative Commons license, and indicate if changes were made.

\section{References}

1. Adolfsson, K., Enelund, M., Olsson, P.: On the fractional order model of viscoelasticity. Mec. TimeDependent Mater. 9(1), 15-34 (2005)

2. Al-Alaoui, M.A.: Al-Alaoui operator and the new transformation polynomials for discretization of analogue systems. Electr. Eng. 90(6), 455-467 (2008)

3. Aldhaheri, R.W.: Model order reduction via real Schur-form decomposition. Int. J. Control. 53(3), 709-716 (1991) 
4. Aldhaheri, R.W.: Design of low order IIR filters with an arbitrary frequency range via frequency domain cross Gramian. In: Proc. of the 1st International Conference on Modeling, Simulation and Applied Optimization (2005)

5. Aldhaheri, R.W.: Frequency-domain model reduction approach to design IIR digital filters using orthonormal bases. AEU - Int. J. Electron. Commun. 60(6), 413-420 (2006)

6. Antoulas, A.: Approximation of Large-Scale Dynamical System. SIAM, Philadelphia (2005)

7. Baranowski, J., Bauer, W., Zagórowska, M., Piatek, P.: On digital realizations of non-integer order filters. Circ. Syst. Signal Process. 35(6), 2083-2107 (2016)

8. Baur, U.: Low rank solution of data-sparse Sylvester equations. Numer. Linear Algebra Appl., 15(9) (2008)

9. Baur, U., Benner, P.: Cross-Gramian based model reduction for data-sparse systems. Electron. Trans. Numer. Anal. 31, 256-270 (2008)

10. Benner, P., Kürschner, P., Saak, J.: Frequency-limited balanced truncation with low-rank approximations. SIAM J. Sci. Comput. 38(1), A471-A499 (2016)

11. Biswas, K., Bohannan, G., Caponetto, R., Mendes Lopes, A., Tenreiro Machado, J.A.: FractionalOrder Devices. SpringerBriefs in Nonlinear Circuits Springer International Publishing (2017)

12. Chen, Y.Q., Moore, K.L.: Discretization schemes for fractional-order differentiators and integrators. IEEE Trans. Circuts Systems I: Fund. Theory Appl. 49(3), 363-365 (2002)

13. Condon, M., Ivanov, R.: Empirical balanced truncation of nonlinear systems. J. Nonlinear Sci. 14(5), 405-414 (2004)

14. Datta, K.: The matrix equation $\mathrm{XA}-\mathrm{BX}=\mathrm{R}$ and its applications. Linear Algebra Appl. 109, 91-105 (1988)

15. Deng, Z., Cao, H., Li, X., Jiang, J., Yang, J., Qin, Y.: Generalized predictive control for fractional order dynamic model of solid oxide fuel cell output power. J. Power. Sources 195(24), 8097-8103 (2010)

16. Enns, D.: Model reduction with balanced realizations: an error bound and frequency weighted generalization. In: 23rd IEEE Conf. on Decision and Control, pp. 127-132 (1984)

17. Fernando, K., Nicholson, H.: On the structure of balanced and other principal representations of SISO systems. IEEE Trans. Autom. Control 28(2), 228-231 (1983)

18. Fernando, K., Nicholson, H.: On the cross-Gramian for symmetric MIMO systems. IEEE Trans. Circ. Syst. 32(5), 487-489 (1985)

19. Gabano, J., Poinot, T.: Fractional modelling and identification of thermal systems. Signal Process. 91(3), 531-541 (2011)

20. Gabano, J.D., Poinot, T., Huard, B.: Bounded diffusion impedance characterization of battery electrodes using fractional modeling. Commun. Nonlinear Sci. Numer. Simul. 47, 164-177 (2017)

21. Garrappa, R., Maione, G.: Model order reduction on Krylov subspaces for fractional linear systems. IFAC Proc. 46(1), 143-148 (2013). 6th IFAC Workshop on Fractional Differentiation and Its Applications

22. Gawronski, W., Juang, J.: Model reduction in limited time and frequency intervals. Int. J. Syst. Sci. 21(2), 349-376 (1990)

23. Gosea, I.V., Petreczky, M., Antoulas, A.C., Fiter, C.: Balanced truncation for linear switched systems. Advances in Computational Mathematics. https://doi.org/10.1007/s10444-018-9610-z (2018)

24. Hahn, J., Edgar, T.F.: An improved method for nonlinear model reduction using balancing of empirical Gramians. Comput. Chem. Eng. 26(10), 1379-1397 (2002)

25. Haider, K.S., Ghafoor, A., Imran, M., Malik, F.M.: Model reduction of large scale descriptor systems using time limited Gramians. Asian J. Control 19(3), 1217-1227 (2017)

26. Himpe, C., Ohlberger, M.: A note on the cross Gramian for non-symmetric systems. Syst. Sci. Control Eng. 4(1), 199-208 (2016)

27. Himpe, C., Ohlberger, M.: Cross-Gramian-based model reduction: a comparison. In: Benner, P., Ohlberger, M., Patera, A., Rozza, G., Urban, K. (eds.) Model Reduction of Parametrized Systems, pp. 271-283. Springer International Publishing (2017)

28. Imran, M., Ghafoor, A., Imran, M.: Frequency limited model reduction techniques with error bounds. IEEE Trans. Circ. Syst. II: Express Briefs 65(1), 86-90 (2018)

29. Imran, M., Ghafoor, A., Sreeram, V.: A frequency weighted model order reduction technique and error bounds. Automatica 50(12), 3304-3309 (2014)

30. Ionescu, C., Lopes, A., Copot, D., Machado, J.A.T., Bates, J.H.T.: The role of fractional calculus in modeling biological phenomena: a review. Commun. Nonlinear Sci. Numer. Simul. 51, 141-159 (2017) 
31. Jazlan, A., Sreeram, V., Shaker, H.R., Togneri, R., Minh, H.B.: Frequency interval cross Gramians for linear and bilinear systems. Asian J. Control 19(1), 22-34 (2017)

32. Jazlan, A., Sreeram, V., Togneri, R.: Cross Gramian based time interval model reduction. In: Australian Control Conference, pp. 139-141 (2015)

33. Jbilou, K.: Low rank approximate solutions to large Sylvester matrix equations. Appl. Math. Comput. 177(1), 365-376 (2006)

34. Jesus, I.S., Tenreiro Machado, J.A.: Fractional control of heat diffusion systems. Nonlinear Dyn. 54(3), 263-282 (2008)

35. Jiang, Y., Xu, K.: H2 optimal reduced models of general MIMO LTI systems via the cross Gramian on the Stiefel manifold. J. Franklin Inst. 354(8), 3210-3224 (2017)

36. Krajewski, W., Viaro, U.: A method for the integer-order approximation of fractional-order systems. J. Franklin Inst. 351(1), 555-564 (2014)

37. Kürschner, P.: Balanced truncation model order reduction in limited time intervals for large systems. Advances in Computational Mathematics. https://doi.org/10.1007/s10444-018-9608-6 (2018)

38. Laub, A., Heath, M., Paige, C., Ward, R.: Computation of system balancing transformations and other applications of simultaneous diagonalization algorithms. IEEE Trans. Autom. Control 32(2), 115-122 (1987)

39. Laub, A.J., Silverman, L.M., Verma, M.: A note on cross-Grammians for symmetric realizations. Proc. IEEE 71(7), 904-905 (1983)

40. Lin, C., Chiu, T.: Model reduction via frequency weighted balanced realization. Theory Adv. Technol. 8, 341-451 (1992)

41. Mansouri, R., Bettayeb, M., Djennoune, S.: Optimal reduced-order approximation of fractional dynamical systems. AIP Conf. Proc. 1019(1), 127-132 (2008)

42. Mashayekhi, S., Miles, P., Hussaini, M.Y., Oates, W.S.: Fractional viscoelasticity in fractal and nonfractal media: Theory, experimental validation, and uncertainty analysis. J. Mech. Phys. Solids 111, 134-156 (2018)

43. Moaveni, B., Khaki-Sedigh, A.: A new approach to compute the cross-Gramian matrix and its application in input-output pairing of linear multivariable plants. J. Appl. Sci. 8(4), 608-614 (2008)

44. Monje, C., Chen, Y., Vinagre, B., Xue, D., Feliu-Batlle, V.: Fractional-Order Systems and Controls: Fundamentals and Applications. Series on Advances in Industrial Control. Springer, London (2010)

45. Moore, B.: Principal component analysis in linear systems: Controllability, observability and model reduction. IEEE Trans. Autom. Control AC-26(1), 17-32 (1981)

46. Oustaloup, A., Levron, F., Mathieu, B., Nanot, F.M.: Frequency-band complex noninteger differentiator: Characterization and synthesis. IEEE Trans. Circ. Syst. I: Fund. Theory Appl. 47(1), 25-39 (2000)

47. Podlubny, I.: Fractional Differential Equations. Academic Press, Orlando (1999)

48. Podlubny, I.: Fractional-order systems and $P I^{\lambda} D^{\mu}$ controllers. IEEE Trans. Autom. Control 44(1), 208-214 (1999)

49. Rydel, M., Stanisławski, R.: A new frequency weighted Fourier-based method for model order reduction. Automatica 88, 107-112 (2018)

50. Rydel, M., Stanisławski, W.: Selection of reduction parameters for complex plant MIMO LTI models using the evolutionary algorithm. Math. Comput. Simul. 140, 94-106 (2017)

51. Sabatier, J., Aoun, M., Oustaloup, A., Grgoire, G., Ragot, F., Roy, P.: Fractional system identification for lead acid battery state of charge estimation. Signal Process. 86(10), 2645-2657 (2006)

52. Safonov, M.G., Chiang, R.Y.: A Schur method for balanced-truncation model reduction. IEEE Trans. Autom. Control 34(7), 729-733 (1989)

53. Shaker, H.R., Tahavori, M.: Control configuration selection for bilinear systems via generalised Hankel interaction index array. Int. J. Control. 88(1), 30-37 (2015)

54. Shen, J., Lam, J.: $H_{\infty}$ model reduction for positive fractional order systems. Asian J. Control 16(2), 441-450 (2014)

55. Sierociuk, D., Dzieliński, A., Sarwas, G., Petras, I., Podlubny, I., Skovranek, T.: Modelling heat transfer in heterogeneous media using fractional calculus. Philos. Trans. R. Soc. London A: Math. Phys. Eng. Sci., 371(1990) (2013)

56. Sopasakis, P., Sarimveis, H.: Stabilising model predictive control for discrete-time fractional-order systems. Automatica 75, 24-31 (2017)

57. Sorensen, D.C., Antoulas, A.C.: The Sylvester equation and approximate balanced reduction. Linear Algebra Appl. 351-352, 671-700 (2002) 
58. Stanisławski, R., Latawiec, K.J.: Fractional-order discrete-time Laguerre filters - a new tool for modeling and stability analysis of fractional-order LTI SISO systems. Discret. Dyn. Nat. Soc., 1-9: Article ID: 9590687 (2016)

59. Stanisławski, R., Latawiec, K.J., Gałek, M., Łukaniszyn, M.: Modeling and identification of a fractionalorder discrete-time SISO Laguerre-Wiener system. In: Proceedings of the 19th International Conference on Methods and Models in Automation and Robotics, pp. 165-168. Miedzyzdroje (2014)

60. Stanisławski, R., Rydel, M., Latawiec, K.J.: Modeling of discrete-time fractional-order state space systems using the balanced truncation method. J. Franklin Inst. 354(7), 3008-3020 (2017)

61. Tahavori, M., Shaker, H.R.: Model reduction via time-interval balanced stochastic truncation for linear time invariant systems. Int. J. Syst. Sci. 44(3), 493-501 (2013)

62. Tavakoli-Kakhki, M., Haeri, M.: Model reduction in commensurate fractional-order linear systems. Proc. Institut. Mech. Eng. Part I: J. Syst. Control Eng. 223(4), 493-505 (2009)

63. Varga, A., Anderson, B.: Accuracy-enhancing methods for balancing-related frequency-weighted model and controller reduction. Automatica 39(5), 919-927 (2003)

64. Vinagre, B.M., Petráš, I., Podlubny, I., Chen, Y.Q.: Using fractional order adjustment rules and fractional order reference models in model-reference adaptive control. Nonlin. Dyn. 29(1), 269-279 (2002)

65. Vinagre, B.M., Podlubny, I., Hernandez, A., Feliu, V.: Some approximations of fractional order operators used in control theory and applications. Fract. Calcul. Appl. Anal. 3(3), 231-248 (2000)

66. Wang, G., Sreeram, V., Liu, W.Q.: A new frequency-weighted balanced truncation method and an error bound. IEEE Trans. Autom. Control 44(9), 1734-1737 (1999)

67. Willcox, K., Megretski, A.: Fourier series for accurate, stable, reduced-order models in large-scale linear applications. SIAM J. Sci. Comput. 26(3), 944-962 (2005)

68. Zulfiqar, U., Imran, M., Ghafoor, A.: Cross-Gramian based frequency-weighted model order reduction technique. Electron. Lett. 52(16), 1376-1377 (2016) 